\title{
Channel cracks in a hermetic coating consisting of organic and inorganic layers
}

\section{Citation}

Cordero, Nicolas, Juil Yoon, and Zhigang Suo. 2007. "Channel Cracks in a Hermetic Coating Consisting of Organic and Inorganic Layers." Applied Physics Letters 90 (11): 111910. https:// doi.org/10.1063/1.2713134.

\section{Permanent link}

http://nrs.harvard.edu/urn-3:HUL.InstRepos:41467479

\section{Terms of Use}

This article was downloaded from Harvard University's DASH repository, and is made available under the terms and conditions applicable to Other Posted Material, as set forth at http:// nrs.harvard.edu/urn-3:HUL.InstRepos:dash.current.terms-of-use\#LAA

\section{Share Your Story}

The Harvard community has made this article openly available.

Please share how this access benefits you. Submit a story.

Accessibility 


\title{
Channel cracks in a hermetic coating consisting of organic and inorganic layers
}

\author{
Nicolas Cordero, Juil Yoon, ${ }^{\text {a) }}$ and Zhigang Suo ${ }^{\text {b) }}$ \\ Harvard School of Engineering and Applied Sciences, Harvard University, Cambridge, \\ Massachusetts 02138
}

(Received 12 January 2006; accepted 8 February 2007; published online 14 March 2007)

\begin{abstract}
Flexible electronic devices often require hermetic coatings that can withstand applied strains. This letter calculates the critical strains for various configurations of channel cracks in a coating consisting of organic and inorganic layers. The authors show that the coating can sustain the largest strain when the organic layer is of some intermediate thicknesses. (C) 2007 American Institute of Physics. [DOI: 10.1063/1.2713134]
\end{abstract}

Flexible electronics is promising for diverse applications, ${ }^{1,2}$ such as rollable displays, conformal sensors, and printable solar cells. These systems are thin, rugged, and lightweight. They can be manufactured at low costs, for example, by roll-to-roll printing. The development of flexible electronics has raised many issues concerning the mechanical behavior of materials. ${ }^{3-6}$ This letter examines a particular issue: channel cracks in hermetic coatings.

Electronic devices (e.g., organic light-emitting devices) often degrade when exposed to air. ${ }^{7,8}$ Developing hermetic coatings has been a significant challenge. Organic films are permeable to gases, and inorganic films inevitably contain processing flaws, so that neither by themselves are effective gas barriers. These considerations have led to the development of multilayer coatings consisting of alternating organic and inorganic films. ${ }^{8-10}$ To be used in flexible electronics, these coatings must also withstand applied strains without forming channel cracks. ${ }^{4,11}$

Channel cracks have been studied in the context of microelectronic devices. ${ }^{12-21}$ Two differences between microelectronic and flexible electronic devices should be mentioned. First, the magnitudes of strain in the two types of devices can be quite different. While thin films in microelectronic devices are principally under residual stresses associated with fabrication processes, flexible electronic devices may experience one-time severe stretch in fabrication or repeated bending and twisting in service. Second, the substrates in the two types of devices are different. While substrates for microelectronic devices are stiff materials such as silicon, substrates for flexible electronic devices are polymers. It is well known that a stiff substrate constrains the opening displacement of a channel crack, but this constraint is substantially reduces when the substrate is compliant. Consequently, the driving force for a channel crack in a film on a compliant substrate is much larger than that on a stiff substrate, with everything else being equal. the organic layers is to decouple processing flaws in the inorganic layers so that the coating acts as an effective gas barrier. To illustrate mechanical issues, we analyze a threelayer coating on a polymer substrate. The coating consists of two inorganic layers sandwiching an organic layer and is

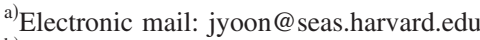

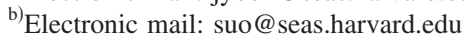

bonded on a polymer substrate. The whole structure is subject to a strain $\varepsilon_{\text {appl }}$ parallel to the layers. When the strain reaches a critical value, a crack may form in the coating. Figure 1 illustrates several cases of cracking. In case (a), a crack channels in the top inorganic layer. Case (b) can be considered as a continuation of case (a), that is, after a crack forms in the top inorganic layer, a new crack tunnels in the bottom inorganic layer. In case (c), a channel crack and a tunnel crack propagate simultaneously in the two inorganic layers. Because a channel crack is easier to propagate than a tunnel crack, case (e) is less critical than case (a) and will not be studied in this letter. Case (d) deals with a channel crack across the entire thickness of the coating.

The aim of this letter is to study how the thickness of the organic layer, $h_{p}$, affects the critical strain. If the organic layer is too thin, it cannot stop the propagation of a crack across the organic/inorganic interfaces. If the organic layer is too thick, the constraint between the two inorganic layers is removed, so that channel cracks can readily form in either inorganic layer. Consequently, we expect that an intermediate thickness of organic layer maximizes the strain sustainable by the coating.

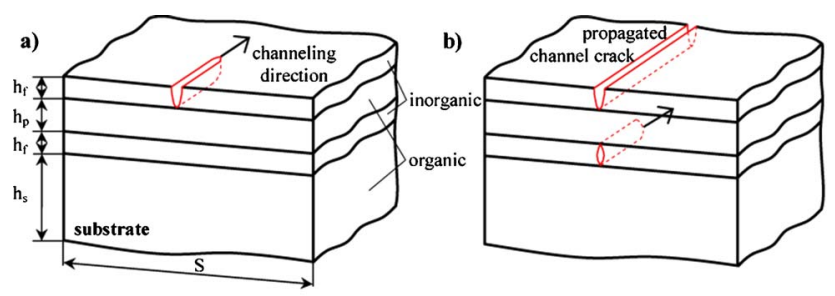

In a multilayer hermetic coating, the main function of c)

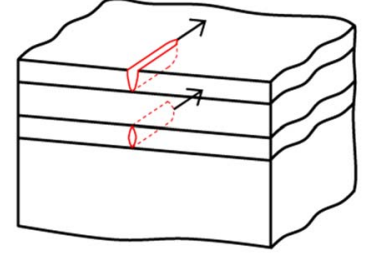

e)

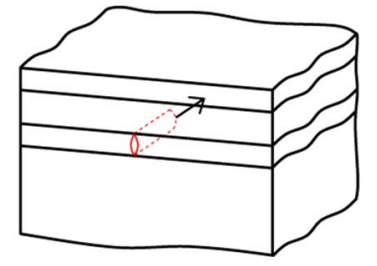

FIG. 1. Several cases of channel cracks in a three-layer coating.

d)

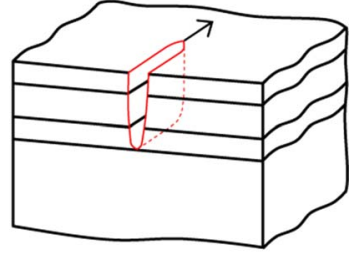

Downloaded 14 Mar 2007 to 128.103.60.225. Redistribution subject to AIP license or copyright, see http://apl.aip.org/apl/copyright.jsp 


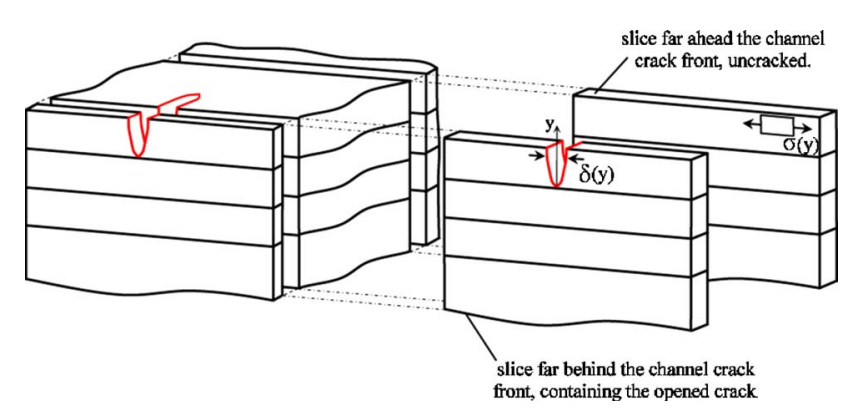

FIG. 2. Reduction in the elastic energy associated with a crack advancing a unit distance, $U$, is calculated from the elastic energy stored in a slice of material of unit thickness far ahead of the crack front minus the elastic energy stored in a slice of material of unit thickness far behind the crack front.

All materials in the structure are taken to be linearly elastic. Let $U$ be the reduction of the elastic energy associated with a crack advancing a unit distance. As illustrated in Fig. 2, this reduction can be calculated by the elastic energy stored in a slice of material of unit thickness far ahead of the crack front minus the elastic energy stored in a slice of material of unit thickness far behind the crack front. This energy reduction is given by ${ }^{12,13}$

$$
U=\frac{1}{2} \int \sigma(y) \delta(y) d y,
$$

where $y$ is the coordinate normal to the layers, $\sigma(y)$ is the stress in the slice far ahead of the crack front, and $\delta(y)$ is the opening displacement in the slice far behind the channel crack front. The stress $\sigma(y)$ and the opening $\delta(y)$ can be obtained by solving two elasticity problems under the plane strain conditions. The integration extends over the thickness of the layer(s) in which the crack forms. That is, the integration extends over the thickness of the top inorganic film in case (a), over the thickness of the bottom inorganic film in case (b) and the thickness of both the inorganic films in case (c), and over the entire thickness of the coating in case (d).

Dimensional considerations dictate that this reduction in energy takes the form

$$
U=E_{f}^{*} \varepsilon_{\mathrm{appl}}^{2} h_{f}^{2} f\left(\frac{h_{p}}{h_{f}}, \frac{E_{p}}{E_{f}}\right) .
$$

Here $E_{f}^{*}=E_{f} /\left(1-\nu_{f}^{2}\right)$ is the plane strain modulus of the inorganic material. We assume that the organic layer and the polymer substrate have identical Young's modulus $E_{p}$. The two inorganic films are taken to be identical, with thickness $h_{f}$ and Young's modulus $E_{f}$. Poisson's ratios $\nu_{f}$ and $\nu_{p}$ are taken to be 0.3 . We fix the ratio $E_{f} / E_{p}=150$ but vary the ratio $h_{p} / h_{f}$. All layers are assumed to be well bonded.

The dimensionless functions $f$ for the various cases in Fig. 1 are designated as $f_{a}, f_{b}, f_{c}$, and $f_{d}$. They are calculated using the finite element code ABAQUS. To model a thin coating on a thick substrate, we set the thickness of the substrate to be $1000 h_{f}$. To model an isolated crack in the coating, we use the periodic boundary conditions in the lateral direction and set the period to be $1000 h_{f}$.

A crack will propagate in the inorganic material if the reduction in the elastic energy $U$ exceeds the fracture energy $\Gamma_{f}$ times the thickness of the layer(s) in which the crack occurs. In cases (a) and (b), the critical condition can be defined as requires a large strain. Consequently, when the organic layer
defined as

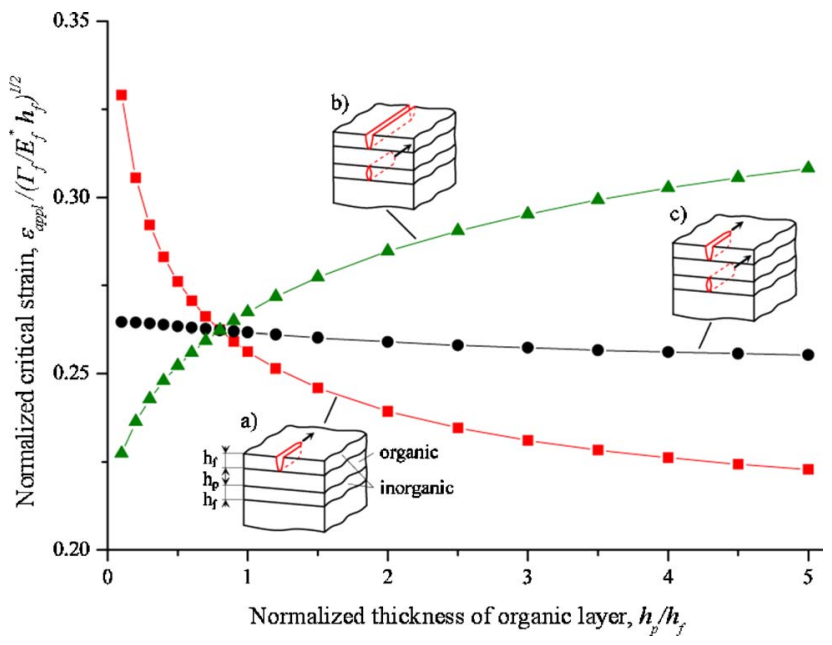

FIG. 3. Normalized critical strain as a function of the normalized thickness of the organic layer for cracks in inorganic layers.

$$
U=h_{f} \Gamma_{f} .
$$

A combination of Eqs. (2) and (3) gives the normalized critical strains

$$
\frac{\varepsilon_{\text {appl }}}{\sqrt{\Gamma_{f} / E_{f}^{*} h_{f}}}=\sqrt{\frac{1}{f_{a}}}
$$

for case (a) and

$$
\frac{\varepsilon_{\text {appl }}}{\sqrt{\Gamma_{f} / E_{f}^{*} h_{f}}}=\sqrt{\frac{1}{f_{b}}}
$$

for case (b).

In case (c), the two cracks propagate in the two inorganic layers simultaneously, so that the critical condition is

$$
U=2 h_{f} \Gamma_{f} .
$$

A combination of Eqs. (6) and (2) gives the following normalized critical strain for case (c):

$$
\frac{\varepsilon_{\text {appl }}}{\sqrt{\Gamma_{f} / E_{f}^{*} h_{f}}}=\sqrt{\frac{2}{f_{c}}} .
$$

Figure 3 plots the calculated normalized critical strains as functions of the normalized layer thickness $h_{p} / h_{f}$. The trends for the three cases are understood as follows. For case (a), the opening of the crack in the top inorganic layer is constrained by the bottom inorganic layer, so that the critical strain decreases as $h_{p}$ increases. By contrast, for case (b), the tunnel crack in the bottom inorganic layer is not constrained by the cracked top inorganic layer but by the substrate and the organic layer, so that the critical strain increases as $h_{p}$ increases. For case (c), the above two effects nearly cancel each other, and the critical strain decreases slowly as $h_{p}$ increases. From the illustration of the three cases (Fig. 1), we note that

$$
f_{b}=f_{c}-f_{a} .
$$

At the ratio $h_{p} / h_{f} \approx 0.8$, the three cases have an identical critical strain because $f_{a}=f_{b}=f_{c} / 2$. When $h_{p} / h_{f}<0.8$, case (b) has the lowest critical strain, but the crack tunnels in the bottom inorganic layer after a channel crack already forms in the top inorganic layer, which corresponds to case (c) and 


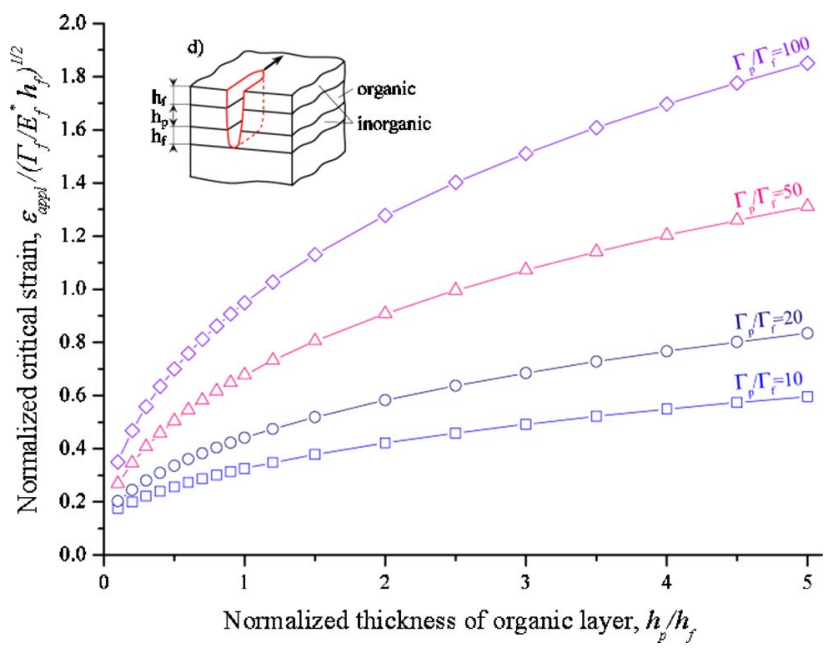

FIG. 4. Normalized critical strain as a function of the normalized thickness of the organic layer for a crack through the entire thickness of the coating.

is thin, case (c) is most critical. When $h_{p} / h_{f}>0.8$, case (a) has the lowest critical strain and is most critical. Thus, in subsequent discussion, we will not consider case (b) and will consider cases (a) and (c).

Now we consider a crack propagating through the whole thickness of the multilayer coating. The channel crack extends when the reduction in the elastic energy equals the total fracture energy:

$$
U=2 h_{f} \Gamma_{f}+h_{p} \Gamma_{p} .
$$

Here $\Gamma_{p}$ is the fracture energy of the organic layer. A combination of the Eqs. (2) and (9) gives the following normalized critical strain:

$$
\frac{\varepsilon_{\mathrm{appl}}}{\sqrt{\Gamma_{f} / E_{f}^{*} h_{f}}}=\sqrt{\frac{2+\left(\Gamma_{p} / \Gamma_{f}\right)\left(h_{p} / h_{f}\right)}{f_{d}}} .
$$

Figure 4 plots the normalized critical strain as a function of the thickness ratio $h_{p} / h_{f}$ at several values of $\Gamma_{p} / \Gamma_{f}$. The critical strain increases as the thickness or the fracture energy of the organic layer increases, with everything else being equal.

Figure 5 summarizes the results for various cases. Case (d) is plotted assuming $\Gamma_{p} / \Gamma_{f}=20$. To avoid cracking, the applied strain should stay in the hatched region. When the organic layer is very thin, it is unable to block the crack from cutting through the interfaces, and the coating fails by a channel crack through the entire thickness. When the organic layer is very thick, the top inorganic layer effectively lies on a compliant substrate, without being constrained by the bottom inorganic layer, and the coating fails by a channel crack in the top inorganic layer. For an organic layer of some intermediate thickness, the coating fails by growing a crack in both inorganic layers, with the organic layer remains intact. Of organic layers of various thicknesses, a layer of intermediate thickness allows the critical strain to attain the maximum value.

As a numerical example, we take the values $\Gamma_{f}=10 \mathrm{~J} \mathrm{~m}^{-2}, \Gamma_{p}=200 \mathrm{~J} \mathrm{~m}^{-2} E_{f}=150 \mathrm{GPa}, E_{p}=1 \mathrm{GPa}$, and $h_{f}=10 \mathrm{~nm}$. We find that the critical strain is $\varepsilon_{\mathrm{cr}}=1.65 \%$ for $h_{p}=1 \mathrm{~nm}, \varepsilon_{\mathrm{cr}}=2.15 \%$ for $h_{p}=5 \mathrm{~nm}$, and $\varepsilon_{\mathrm{cr}}=1.95 \%$ for $h_{p}=20 \mathrm{~nm}$. It is well known, as evident from the dimensional considerations [Eqs. (4), (5), (7), and (10)], that a coating can sustain a large applied strain if the inorganic layers are thin, with everything else being fixed.

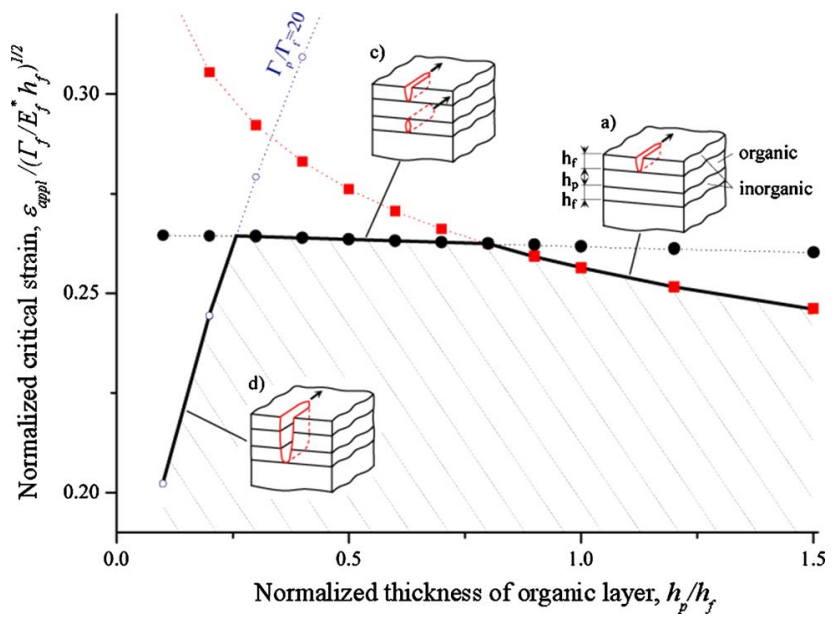

FIG. 5. Comparison of the critical strains for several cases. The hatched area is the safe region, i.e., with no crack propagation in the coating. An optimal thickness exists [in the part of the safe region bounded by the normalized critical strain for case (c)], for which the multilayer coating can sustain the largest applied strain.

In this letter, to illustrate various configurations of channel cracks, we have used a three-layer coating and specified ratios of material properties. The specific values used are representative of an inorganic material (e.g., silicon nitride) and of a polymer (e.g., polyimide). The trends discussed in this letter are expected to be more generally applicable. A systematic parametric study is planed for a subsequent work.

This work was supported by the National Science Foundation. One of the authors (J.Y.) was supported by a postdoctoral fellowship by the Canadian Government.

${ }^{1}$ G. P. Crawford, Flexible Flat Panel Displays (Wiley, Hoboken, NJ, 2005).

${ }^{2}$ A. Nathan and B. R. Chalamala, Proc. IEEE 93, 1235 (2005), special issues on flexible electronics technology.

${ }^{3}$ Z. Suo, J. J. Vlassak, and S. Wagner, China Particuol. 3, 321 (2005).

${ }^{4}$ Y. Leterrier, Prog. Mater. Sci. 48, 1 (2003).

${ }^{5}$ S. P. Lacour, T. Li, D. Chan, S. Wagner, and Z. Suo, Appl. Phys. Lett. 88, 204103 (2006)

${ }^{6}$ Y. Sun, W. M. Choi, H. Jiang, Y. Y. Huang, and J. A. Rogers, Nature Nanotechnology 1, 201 (2006).

${ }^{7}$ H. Aziz, Appl. Phys. Lett. 72, 2642 (1998).

${ }^{8}$ B. D. Vogt, H. J. Lee, V. M. Prabhu, D. M. DeLongchamp, E. K. Lin, and W. L. Wu, J. Appl. Phys. 97, 114509 (2005).

${ }^{9}$ J. S. Lewis and M. S. Weaver, IEEE J. Sel. Top. Quantum Electron. 10, 45 (2004).

${ }^{10}$ A. B. Chwang, M. A. Rothman, S. Y. Mao, R. H. Hewitt, M. S. Weaver, J. A. Silvernail, K. Rajan, M. Hack, J. J. Brown, X. Chu, L. Moro, T. Krajewski, and N. Rutherford, Appl. Phys. Lett. 83, 413 (2003).

${ }^{11}$ G. Rochat, Y. Leterrier, P. Fayet, and J.-A. E. Månson, Thin Solid Films 484, 94 (2005).

${ }^{12}$ J. L. Beuth, Int. J. Solids Struct. 29, 1657 (1992).

${ }^{13}$ J. W. Hutchinson and Z. Suo, Adv. Appl. Mech. 29, 63 (1992).

${ }^{14}$ S. Ho and Z. Suo, J. Appl. Mech. 60, 890 (1993).

${ }^{15}$ J. M. Ambrico and M. R. Begley, Thin Solid Films 419, 144 (2002).

${ }^{16}$ J. J. Vlassak, Int. J. Fract. 119, 299 (2003).

${ }^{17}$ R. Huang, J. H. Prévost, Z. Y. Huang, and Z. Suo, Eng. Fract. Mech. 70, 2513 (2003).

${ }^{18}$ Q. Ma, J. Mater. Res. 12, 840 (1997).

${ }^{19}$ J. He, G. Xu, and Z. Suo, Proceedings of the Seventh International Workshop on Stress-Induced Phenomena in Metallization, Austin, TX, 2004, Vol. 741, pp. 3-14.

${ }^{20}$ T. Y. Tsui, A. J. McKerrow, and J. J. Vlassak, J. Mater. Res. 20, 2266 (2005).

${ }^{21}$ X. H. Liu, M. W. Lane, T. M. Shaw, E. G. Liniger, R. R. Rosenberg, and D. C. Edelstein, Advanced Metallization Conference 2004, San Diego, CA, pp. 361-367. 